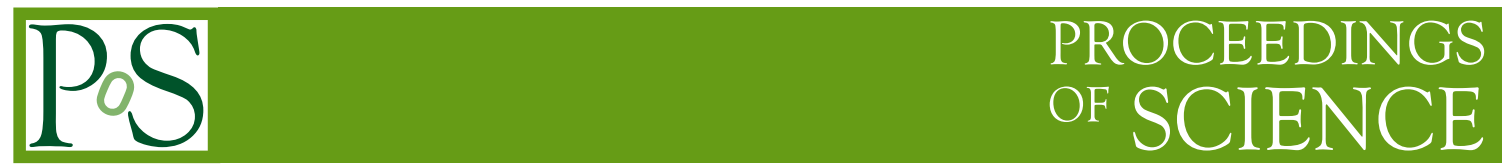

\title{
Cosmic Rays: air showers from low to high energies - Rapporteur Report
}

\author{
Valerio Verzi* \\ Sezione INFN di Roma "Tor Vergata", Roma, Italy \\ E-mail: valerio.verzi@roma2.infn.it
}

This paper summarizes the contributions presented at the 34th International Cosmic Ray Conference related to the cosmic rays detected through the measurement of atmospheric showers. The summary includes all energies, from $\mathrm{TeV}$ up to the end of the energy spectrum at $\sim 10^{20} \mathrm{eV}$.

The 34th International Cosmic Ray Conference,

30 July- 6 August, 2015

The Hague, The Netherlands

${ }^{*}$ Speaker. 


\section{Introduction}

This paper summarizes the contributions on cosmic rays presented at the 34th International Cosmic Ray Conference (ICRC) held in The Hague (The Netherlands) in August 2015. The summary includes all the works in which the cosmic rays are studied by detecting the atmospheric showers, in 8 decades of energies, from $\mathrm{TeV}$ up to the very end of the energy spectrum at $\sim 10^{20}$ $\mathrm{eV}$.

As happened at the previous ICRC conferences, the field tagged as Cosmic Rays (CR) is characterized by a very large number of contributions, demonstrating an extraordinary vitality in the scientific community. According to the conference website ${ }^{1}$, out of a total of 1218 contributions presented at the conference, 460 are tagged as CR with 3 reviews and 6 highlight talks, 143 oral presentations and 306 posters. The 460 contributions are subdivided into three categories: 114 are tagged as CR-EX (Experiment), 117 as CR-IN (Instrumentation) and 75 as CR-TH (Theory). The $460 \mathrm{CR}$ contributions include direct measurements from space which are reported in [1]. The number of contributions related to the detection of air showers is about 300 .

This paper is organized in the following way. In Sec. 2 we report the measurements of the energy spectrum, in Sec. 3 the measurements of the mass composition and in Sec. 4 the results of the searches of anisotropies in the distribution of arrival directions of the cosmic rays. Sec. 5 deals with measurements important to understand the models of the hadronic interactions used in the simulation of extensive air showers. The current status of the radio detection technique and of the future experiments and planned upgrades of the existing ones are described in Sec. 6 and 7 respectively. In the last section we make a short summary of the main achievements.

\section{The energy spectrum}

The measurement of the energy spectrum of the primaries is certainly the main issue for highenergy cosmic ray physics [2]. The spectrum is well described by a power law with an index which is a function of energy and primary mass.
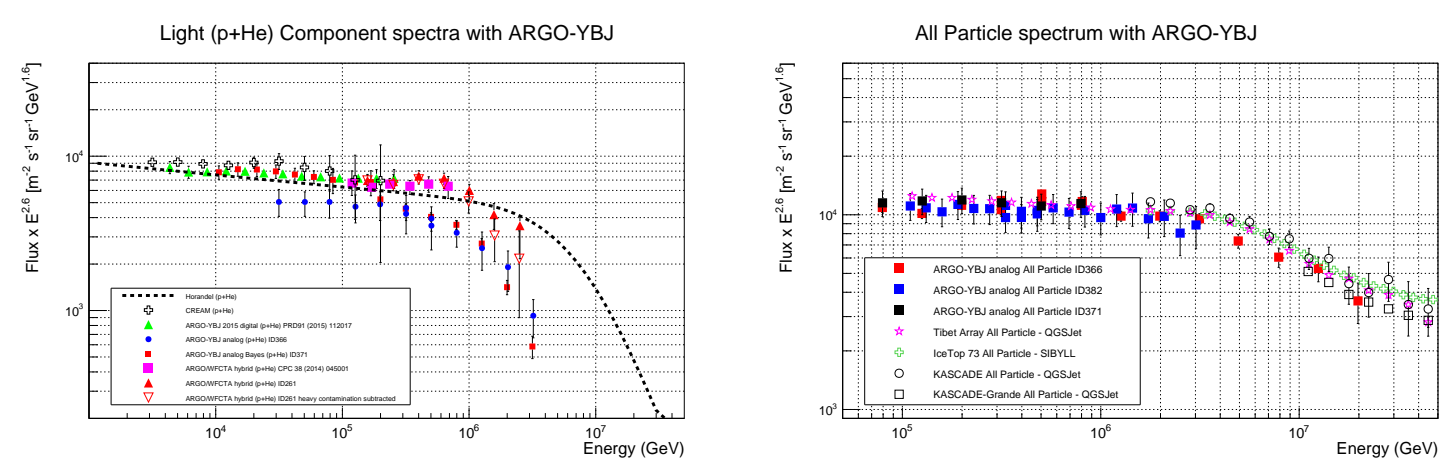

Figure 1: p/He and all-particle energy spectra presented by the ARGO-YBJ collaboration [3, 4, 5]. The figures are taken from [6].

The ARGO-YBJ collaboration has reported a strong evidence for a bending of the $\mathrm{p} / \mathrm{He}$ spectrum below $10^{15} \mathrm{eV}$, at about $700 \mathrm{TeV}[3,4,5]$. In the ARGO-YBJ analysis, the selection of light

\footnotetext{
${ }^{1}$ https://indico.cern.ch/event/344485/contributions
} 
primaries is achieved by exploiting the analog readout of the RPC which allows one to study the particle density distribution up to few meters from the core $[4,5]$ and combining the RPC data with the ones from a wide field of view Cherenkov telescope [3]. The p/He energy spectra are shown in the left panel of Figure 1. The steepening below $10^{15} \mathrm{eV}$ is rather evident. The good agreement with the direct measurements from space is noteworthy. Also the all-particle spectrum obtained with the same analysis techniques agrees well with the measurements from other experiments, with the knee located at $3 \times 10^{15} \mathrm{eV}$ (see the right panel of Figure 1).

The bending of the $\mathrm{p} / \mathrm{He}$ spectrum at $\sim 700 \mathrm{TeV}$ is certainly an important result since it is in tension with the paradigm that the two knees of the energy spectrum at $3 \times 10^{15} \mathrm{eV}$ and at $\sim 10^{17} \mathrm{eV}$ are related to the maximum acceleration energy of the sources or the maximum energy for Galactic magnetic confinement. These grow linearly with the primary charge, with the first knee interpreted as a drop out of the proton component of the flux. It is very important for the ARGO-YBJ results to be confirmed by other experiments.

A confirmation of the ARGO-YBJ results could be provided by the Tibet AS $\gamma$ collaboration that has developed a new hybrid experiment with the aim of measuring the energy spectra of single elements in the energy range from $50 \mathrm{TeV}$ to $10 \mathrm{PeV}$ [7]. Another confirmation could come from
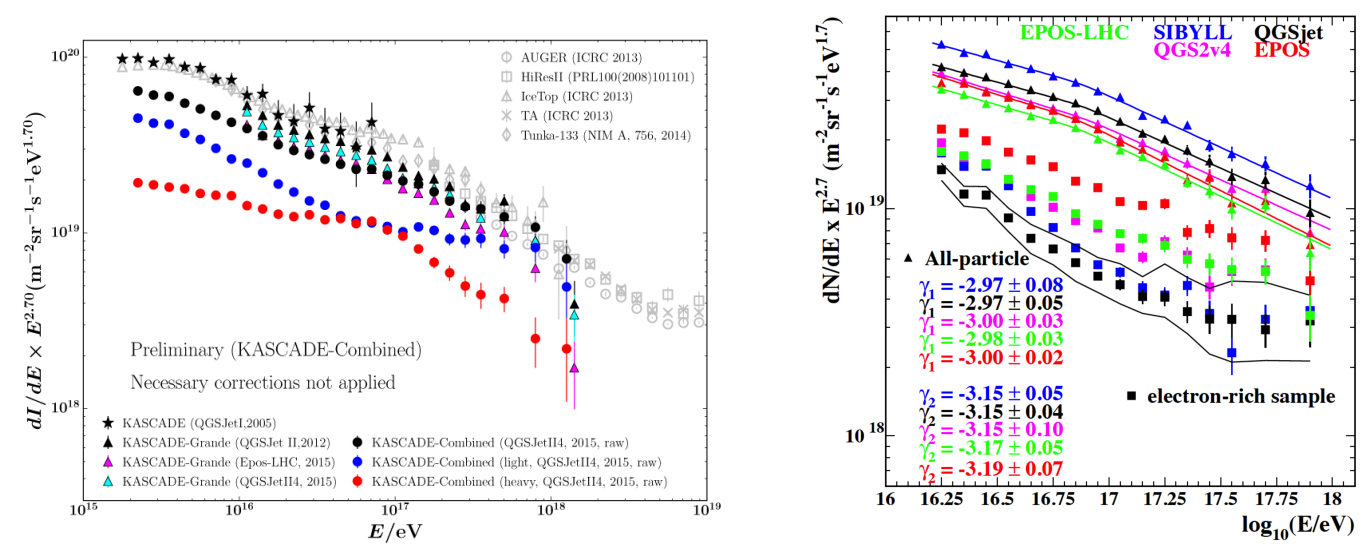

Figure 2: The preliminary energy spectrum obtained with a combined analysis of the KASCADE and KASCADE-Grande array [8, 9] (left) and KASCADE-Grande energy spectrum for different hadronic interaction models [10] (right).

the results of the combined analysis of the KASCADE and KASCADE-Grande arrays that allows measurements to be made in a wide energy range, even from below $10^{15} \mathrm{eV}$ up to $10^{18} \mathrm{eV}$. A very preliminary energy spectrum obtained using this analysis is shown in the left panel of Figure 2 [8, 9]. Careful attention needs to be addressed to the uncertainties arising from the hadronic interaction models used to interpret the data. A study of the impact on the energy spectrum measured with the KASCADE-Grande array of the new interaction models tuned with the LHC data has been discussed in [10]. The KASCADE-Grande energy spectra for different hadronic interaction models are shown in the right panel of Figure 2.

Before the second knee at $\sim 10^{17} \mathrm{eV}$, the energy spectrum manifests a flattening at about $10^{16} \mathrm{eV}$, a feature often called low energy ankle. These features are clearly evident in the energy spectra measured by the Yakutsk and IceCube collaborations (see Figure 3). The Yakutsk spectrum is based on 20 years of continuous operation of the Cherenkov telescopes (1994-2014) [11]. The 
IceCube spectra $[12,13]$ are obtained from events collected by the surface array IceTop (red points) and ones from the coincident events detected by IceTop and the deep-ice detector (black points).
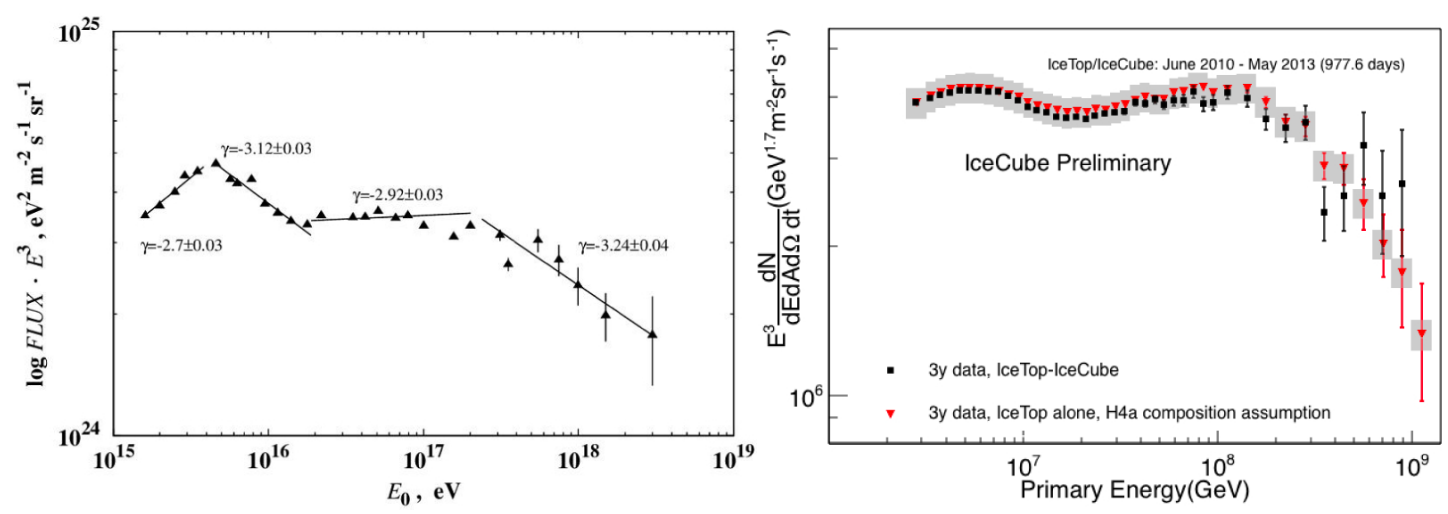

Figure 3: Energy spectra measured with the Small Cherenkov Array in Yakutsk [11] (left) and by the IceCube collaboration [12, 13] (right).

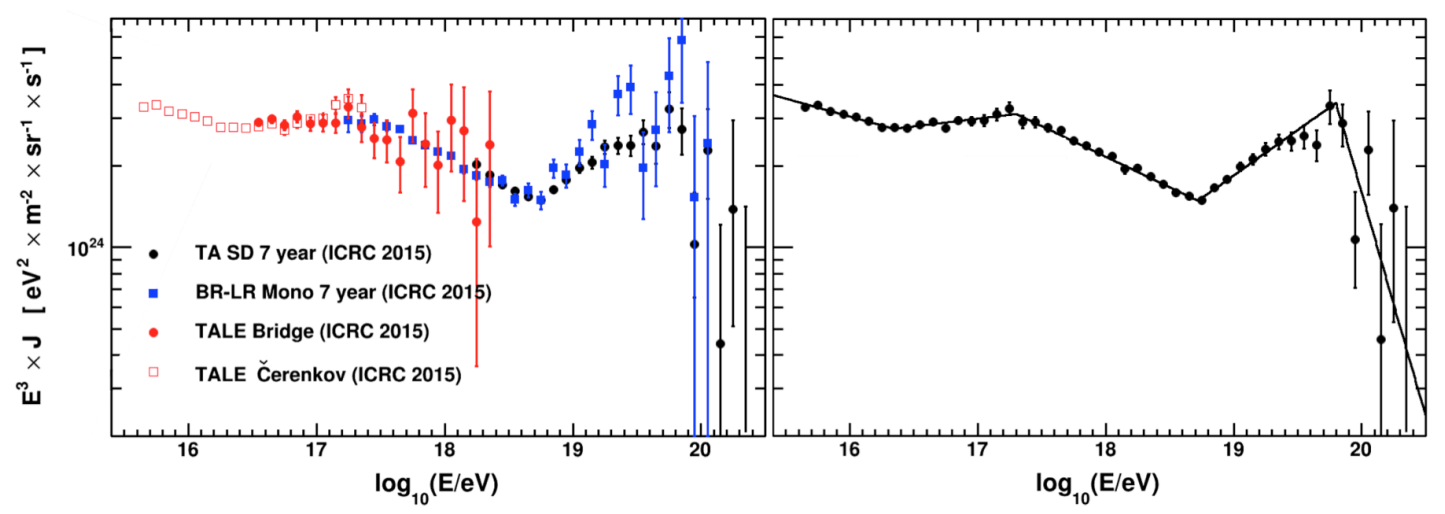

Figure 4: Energy spectra presented by the TA collaboration [16, 19]

The existence of the low energy ankle is confirmed also by the Telescope Array (TA) collaboration with the TA low energy extension (TALE) fluorescence detectors. These telescopes have high elevation viewing angles allowing the detection of nearby low energy showers. TA has developed a reconstruction technique of the events dominated by Cherenkov light which has allowed it to measure the energy spectrum down to energies close to the knee [14]. The TALE events, in which the fluorescence light dominates, are used to extend the spectrum at higher energies [15]. All the energy spectra measured by TA are shown in the left panel of Figure 4. The ground array, which extends over $700 \mathrm{~km}^{2}$, is used to cover the highest energy range [16], while the standard fluorescence telescopes cover the intermediate energies between the ones covered by the surface array and TALE $[17,18]$. The energy spectrum obtained by combining the four spectra is shown in the right panel of Figure $4[16,19]$. The total exposure is about $6,300 \mathrm{~km}^{2} \mathrm{sr}$ yr. Besides the low energy ankle and the second knee, the well known ankle at $\sim 5 \times 10^{18} \mathrm{eV}$ and the break at $\sim 6 \times 10^{19} \mathrm{eV}$ are evident in the TA data.

The energy spectrum at the highest energies is measured with an unprecedented precision at the Pierre Auger Observatory with an exposure exceeding 50,000 $\mathrm{km}^{2} \mathrm{sr}$ yr [20, 21]. The spectrum 

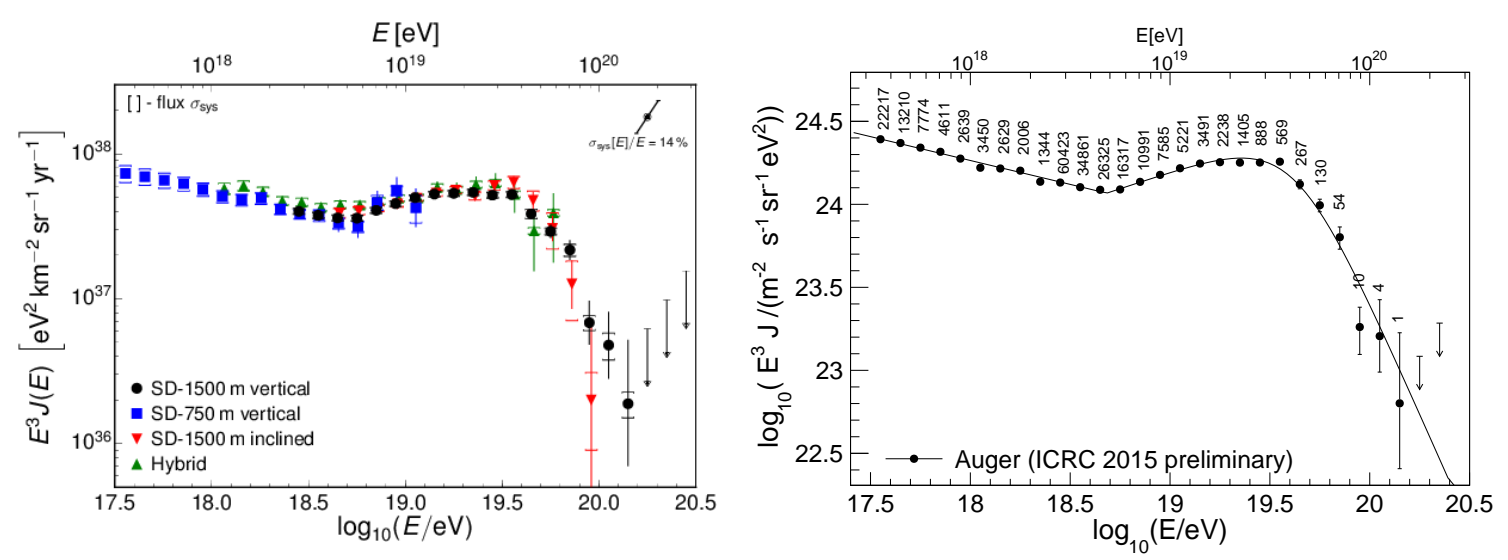

Figure 5: Energy spectra presented by the Auger collaboration [20, 21].

is obtained from the data of the $3000 \mathrm{~km}^{2}$ surface detector (SD) array, the hybrid events detected simultaneously by the fluorescence telescopes and the surface array, and the data from a denser array called Infill, which allows the extension of the sensitivity down to $3 \times 10^{17} \mathrm{eV}$. The SD data are divided into sets according the zenith angle of the showers. The four spectra are shown in the left panel of Figure 5. The energy spectrum obtained combining the four measurements is shown in the right panel.

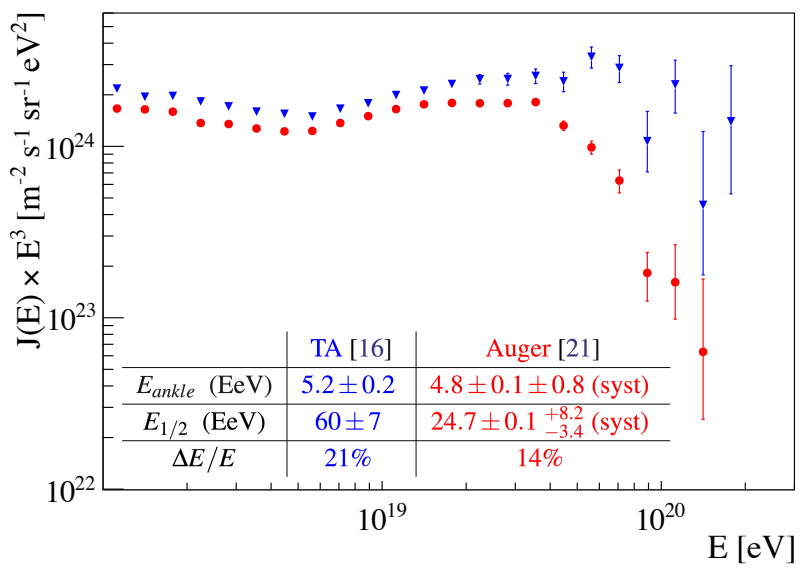

Figure 6: Comparison between the TA [16] (blue) and Auger [21] (red) energy spectra.

As for TA, the ankle and the cut-off at the very end of the spectrum are evident in the Auger data. The TA and Auger combined spectra are compared in Figure 6. While the two measurements are in good agreement at energies below $2 \times 10^{19} \mathrm{eV}$ with a difference in the flux of about $20 \%$, in the region of the cut-off the discrepancy is very significant. A more quantitative statement could be done comparing the position of the ankle $\left(E_{\text {ankle }}\right)$ and of the cut-off $\left(E_{1 / 2}\right)^{2}$ reported by the two collaborations. The systematic uncertainties quoted by Auger include the contribution from the uncertainty in the energy scale. For TA only the statistical error is reported. The uncertainties on the energy $(\Delta E / E)$ are well under control in both experiments since they estimate the shower

\footnotetext{
${ }^{2} E_{1 / 2}$ is the energy at which the integral flux drops to half of what would be expected with no cut-off [22].
} 
energy using the calorimetric measurements of the fluorescence telescopes. As shown in Figure 6, the values of $E_{\text {ankle }}$ are in good agremeent while there is a very significative difference for $E_{1 / 2}$. A common effort by the two collaborations is going on to study the origin of this difference [23].

\section{The mass composition}

The mass composition of cosmic rays is a key quantity in understanding the origin of these particles. Unfortunately this quantity cannot be measured directly but has to be inferred from the measurement of the properties of the atmospheric showers, an inference affected by the large systematic uncertainties related to the hadronic interaction models.
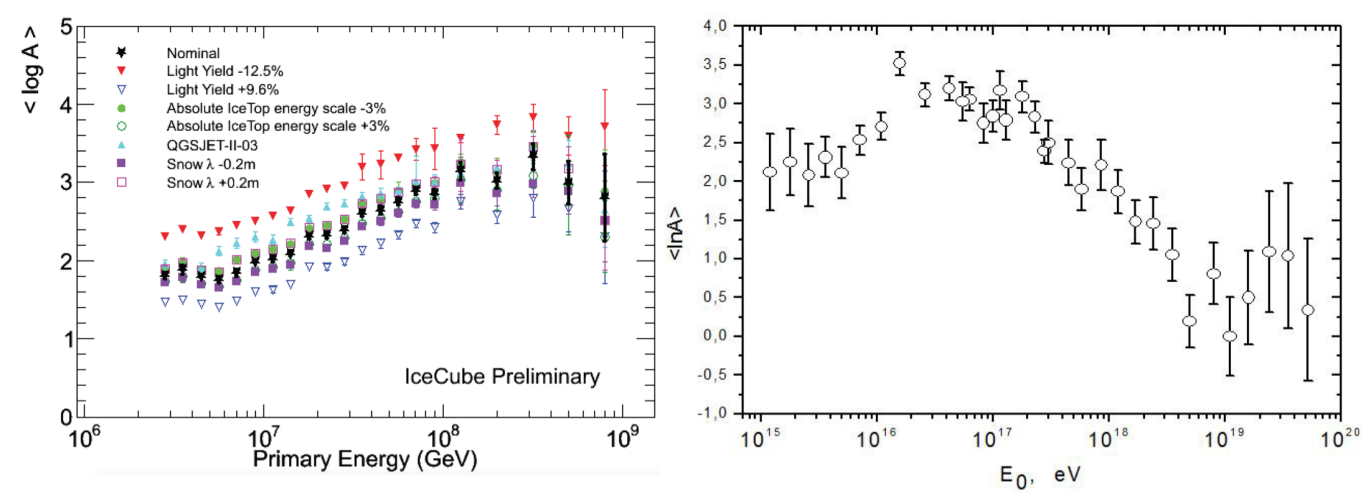

Figure 7: Composition measurements presented by the IceCube collaboration [13] (left) and obtained with the Small Cherenkov Array in Yakutsk [24] (right).

The mean value of logarithm of the mass number $<\ln \mathrm{A}>$ at energies below $10^{17} \mathrm{eV}$ has been measured by the IceCube collaboration using coincident events in IceTop and the deep-ice detector [13], and with the Small Cherenkov Array in Yakutsk [24]. The measurements are shown in Figure 7 and both confirm that the composition becomes heavier from a few times $10^{15} \mathrm{eV}$ to $10^{17}$ $\mathrm{eV}$. Above $10^{17} \mathrm{eV}$ the Yakutsk data shows a clear change of the trend toward lighter masses.

At the highest energies the mass composition is inferred from the measurement of the longitudinal shower profile performed with fluorescence telescopes. The Auger collaboration has presented for the first time an analysis which includes the data of the High Elevation Auger Telescopes (HEAT) that, similarly to the TALE fluorescence telescopes, allows one to observe nearby low energy showers. The events are collected in hybrid mode and the selection criteria are based on geometrical cuts allowing the sampling of almost unbiased distributions of the position of the maximum shower development $X_{\max }$. The mean value of $X_{\max }$ as a function of the shower energy is shown in Figure 8 [25]. The data are compared with the predictions of three hadronic interaction models, two of them (EPOS-LHC and QGSJetII-04) tuned with the LHC data. The $<\ln \mathrm{A}>$ calculated using QGSJetII-04 is shown in the same figure. The Auger data confirm the trend of a mass composition which becomes lighter above $10^{17} \mathrm{eV}$ and a change of the trend at an energy close to the one of the ankle of the energy spectrum, with a composition which becomes heavier at the highest energies.

The Telescope Array collaboration has presented an update of the $X_{\max }$ measurement using different reconstruction techniques. The hybrid events are divided into two sets, one detected by 

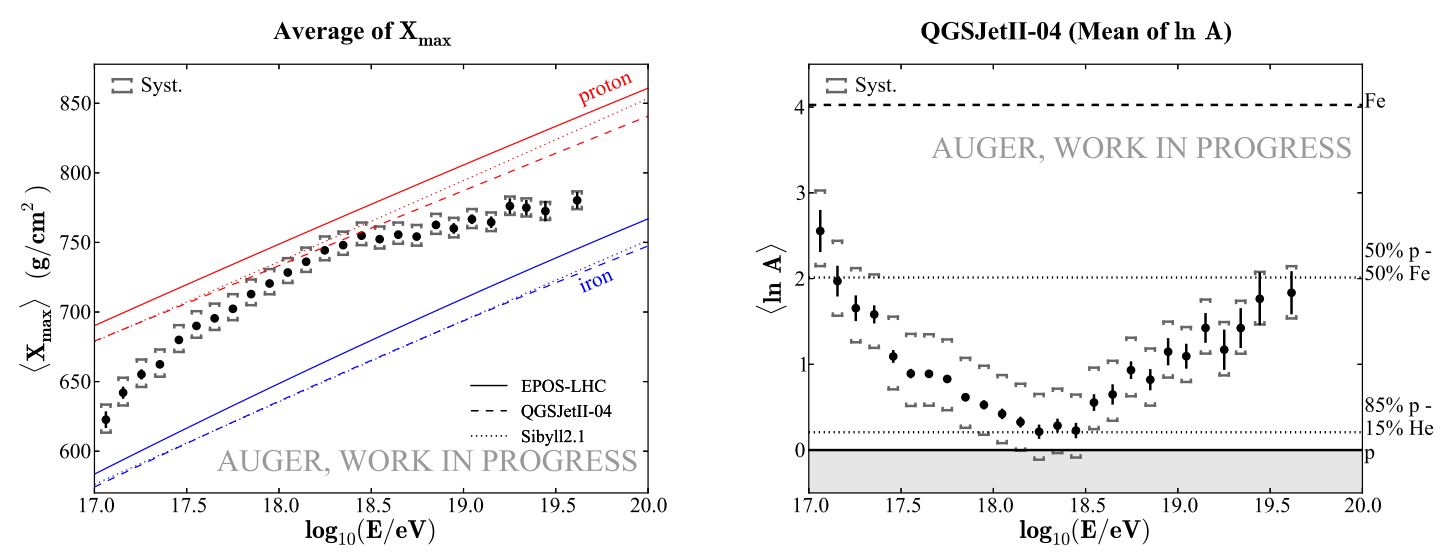

Figure 8: Composition measurements presented by the Auger collaboration [25].

the Middle Drum fluorescence telescope [26] and the other one by the Black Rock and Long Ridge telescopes [18]. For the Middle Drum events a new track finding pattern recognition technique that improves the $X_{\max }$ resolution has been developed [27]. The analysis of the fluorescence events detected by two or more fluorescence telescopes (stereo mode) is described in [28] while the one using the information of only one telescope (mono mode) is presented in [17]. The mean $X_{\max }$ derived from the Middle Drum in hybrid mode is shown in the left panel of Figure 9. It is worth noting that, contrary to Auger, the TA collaboration shows the $X_{\max }$ distributions always folded with the detector effects such as the selection efficiency and acceptance, and therefore biased by experimental effects ${ }^{3}$. The interpretation of the data is performed using Monte Carlo predictions folded with the same detector efficiency and resolution.
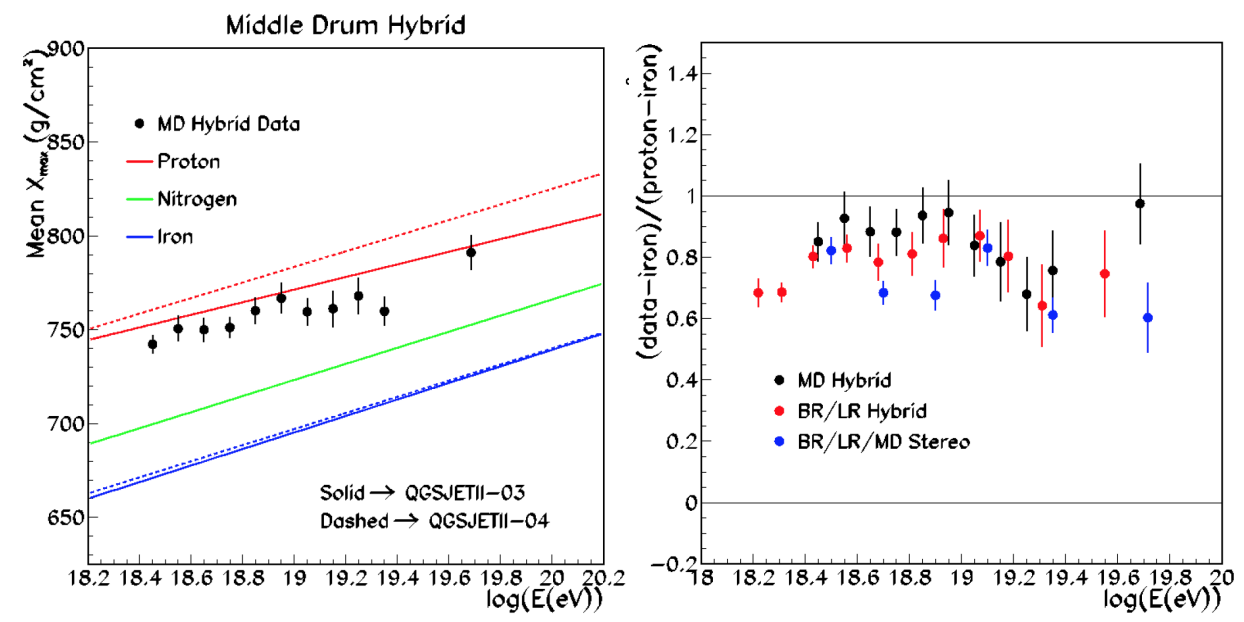

Figure 9: Composition measurements presented by the TA collaboration [29].

Each reconstruction technique has its own acceptance and therefore it is affected by its own systematic uncertainties. The analyses are compared in the right panel of Figure 9 [29]. The plot shows the relative difference in $\left\langle X_{\max }\right\rangle$ between data and protons normalized to the difference

\footnotetext{
${ }^{3}$ with the exception of the mono analysis [17] which uses geometrical cuts similar to the ones used in Auger.
} 
between protons and iron. There is a general consistency among the different analyses demonstrating a good control of the systematic uncertainties. According to QGSJet-II.3, the hadronic model used to calculate $\left\langle X_{\max }\right\rangle$ for protons and iron, all analyses favour a light composition, below the mass of the CNO group. A heavier composition would be obtained using the QGSJet-II.4 model. In fact, as shown in the figure on the left panel, after the tuning with the LHC data the QGSJet model predicts higher values of $X_{\max }$ than before.

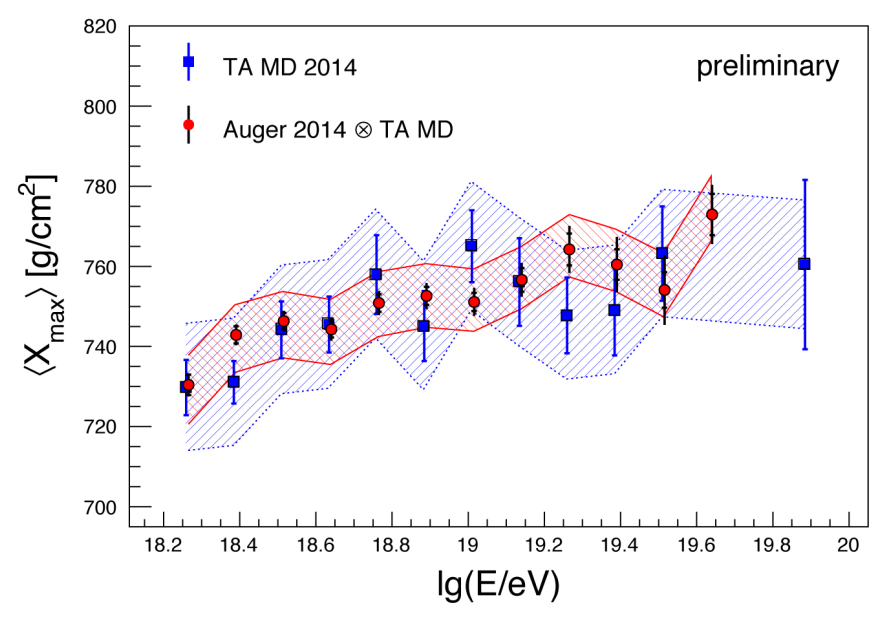

Figure 10: Comparison between the TA data on $X_{\max }$ (blue) and the results obtained on fully simulated TA events whose mass composition is compatible with the $X_{\max }$ distributions measured by Auger (red) [30].

A common effort of the Auger and TA collaborations has been to compare the results on $X_{\max }$ [30]. Due to the different way of presenting the data (unbiased for Auger and folded with detector effects for TA) and the different hadronic interaction models used for the interpretation, the comparison is not straightforward. For that reason the following analysis has been developed. TA events with a mass composition compatible with the $X_{\max }$ distributions measured by Auger have been simulated and reconstructed using the same analysis used for the data. The reconstructed $<X_{\max }>$ for these simulated events is then compared to the measured one (Figure 10), demonstrating a good agreement within the systematic uncertainties.

\subsection{Implications of the mass composition measurements in the interpretation of the energy spectrum}

A correct interpretation of the features observed in the energy spectrum at the highest energies requires the knowledge of the mass composition.

First of all, the absence of photons in the flux of cosmic rays allows one to put very strong limits on the so-called top-down models, in which the cosmic rays are generated by decay of super heavy dark matter or topological defects or similar exotic particles. The updated photon limits presented by Auger [31] are shown in Figure 11. The limits by TA [32] using a new multivariate analysis, but that does not include the systematic uncertainties, are shown in the same figure. Another analysis based on TA hybrid events is presented in [33]. At lower energies new limits from KASCADE are reported in [34]. Other exotic scenarios are excluded by the Auger limits on the neutrino flux [31] and by the limits on ultra-relativistic magnetic monopoles obtained with the events detected by the Auger fluorescence telescopes [35]. 


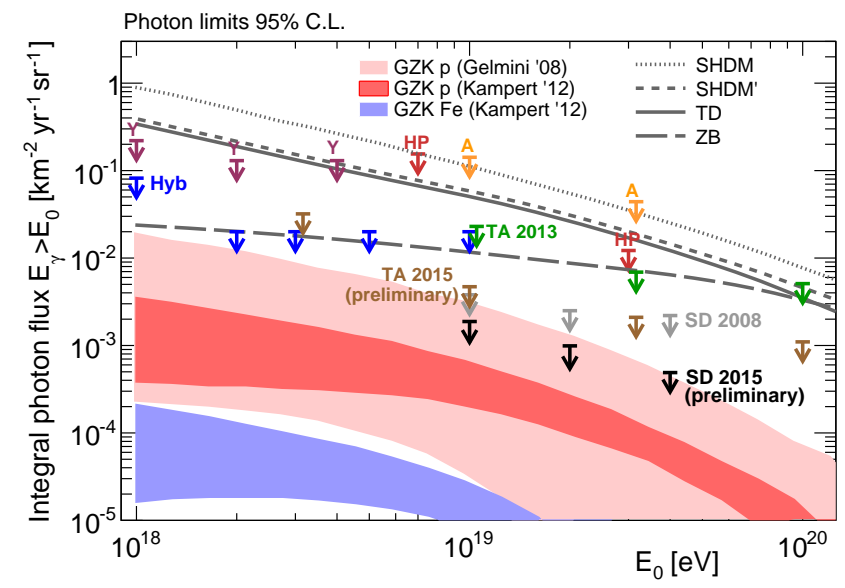

Figure 11: Auger (SD 2015) [31] and TA (TA 2015) [32] new limits on the flux of photons.

Having excluded the top-down models, the cosmic rays have to be of astrophysical origin. Both Auger and TA collaborations have presented analyses aimed at discriminating the most likely astrophysical scenario that is in agreement with their measurements of the flux and of the mass composition.

In the Auger analysis [36], the model is an isotropic distribution of identical sources in a comoving volume where the nuclei are accelerated with a rigidity dependent mechanism. The best fit to the Auger data is a source with hard injection spectra and relatively low maximum injection energies. This would mean that the suppression of the flux at the highest energies would be essentially due by the maximum energies at which the cosmic rays are accelerated, and not to their interaction with the photons of the cosmic microwave background (CMB) radiation, that is the well known GZK effect [37]. There is also a less significative minimum of the fit with rather high maximum injection energies that would favour a photo-disintegration scenario.

The model tested by Auger is limited to energies above $5 \times 10^{18} \mathrm{eV}$ and therefore it does not describe the ankle. A possible interpretation of the ankle and the suppression has been presented by TA [38]. Contrary to Auger, TA assumes that only protons are accelerated with very high maximum injection energies. The sources are distributed either uniformly or following the largescale structure distribution. The conclusion is that the TA spectrum is well described by a strong evolution of sources with redshift within the so called "dip" scenario [39], in which the ankle is due to the interaction of protons with the $\mathrm{CMB}$ photons, with consequent production of $e^{+} e^{-}$pairs and with the GZK cut-off.

The interpretations of the features of the energy spectrum provided by Auger and TA are very different and in tension between them. A pure proton composition seems to be excluded by the $X_{\max }$ data of Auger. Moreover the Auger collaboration has presented an analysis aiming to estimate the mass composition using both $X_{\max }$ and the signals measured by the surface array [40]. This analysis suggests that the composition at the ankle is mixed, with a significative mass dispersion, a result that is in tension with the high fraction of protons required to explain the ankle with electron-positron pair production [41].

Other models which include an interpretation of the ankle have been presented. In [43] the 
model describes the evolution of the cosmic ray spectrum and mass composition with energy using on only two components, one Galactic depending on rigidity alone and another one extragalactic. In [44] the spectrum and the composition of the extragalactic component, which is assumed to dominate above the second knee, are accounted by the photodisintegration of the nuclei in the region surrounding a cosmic ray accelerator.

\section{Anisotropy}

Results on the anisotropy of the arrival direction distribution of cosmic rays at $\mathrm{TeV}$ energies in the Northern Hemisphere has been presented by the HAWC collaboration [45]. The analysis uses data collected during the operation of about one third of the water-Cherenkov detectors, in a period of 181 days and for a total of 85.6 billion events. The relative intensity of the cosmic ray flux after fit and subtraction of the lowest order moments, shows excesses corresponding to the so called Regions A, B and C previously observed by other experiments. The strongest excess is for region A at ( $\alpha=60^{\circ}, \delta=10^{\circ}$ - equatorial coordinates), which has been observed to have an energy spectrum harder than the one obtained in off-source regions.

Results on anisotropy in the Southern Hemisphere between $\mathrm{TeV}$ and $\mathrm{PeV}$ energies have been reported by the IceCube collaboration [46]. The data sample consists on 250 billion events recorded over a period of 5 years. The small-scale structure of the cosmic ray flux after the subtraction of the dipole and quadrupole moments has been shown. The large-scale structure has been studied as a function of the energy. The amplitude of the dipole fit decreases steadily up to $\sim 200 \mathrm{TeV}$, then increases up to $5 \mathrm{PeV}$. The dipole phase shifts rather abruptly above $100 \mathrm{TeV}$, from a large-scale maximum around $50^{\circ}$ to $200^{\circ}$ in right ascension. IceCube has also reported the first $\mathrm{PeV}$ neutron flux limits for the Southern sky [47].

The HAWC and IceCube collaborations have started a common effort to make a joint analysis of the anisotropy [48]. Currently the HAWC statistics are too suppressed when the datasets of the two experiments are restricted to compatible energies and new data are required to better control the systematic uncertainties.

The Tibet AS $\gamma$ collaboration has reported the observation of the large-scale sidereal anisotropy of cosmic rays based on a 15 years data taking of the Tibet Air Shower experiment [49]. The energy dependencies of the first harmonic coefficients of the large-scale anisotropy indicates a change of the anisotropy between 10 and $1000 \mathrm{TeV}$. A full-sky picture of the large-scale anisotropy using the Tibet Air Shower and IceCube experiments has been presented in [49, 50].

Motivated by the bending of the $\mathrm{p}+\mathrm{He}$ energy spectrum below $10^{15} \mathrm{eV}$, the ARGO-YBJ collaboration has presented a new approach to measure the anisotropy for different primary particle masses [51].

At higher energies, the study of the large-scale anisotropy is important since it could provide information at which energies the flux of extragalactic cosmic rays starts to dominate the flux of galactic ones. The Auger collaboration has reported the current status of a prescribed test on the phase of the first harmonic modulation in right ascension [52]. In previous publications there was a hint of a smooth phase transition from $270^{\circ}$ to $100^{\circ}$ at about $10^{18} \mathrm{eV}$. At almost the end of the prescription the phase alignment is rather uncertain suggesting that the prescription will be not fulfilled. 

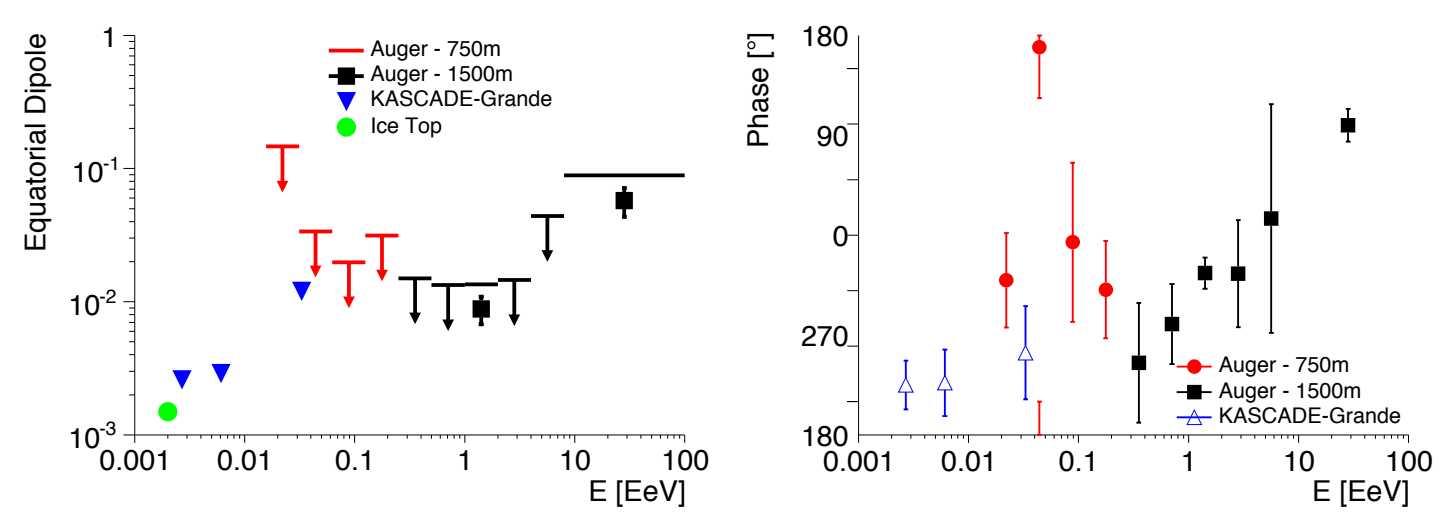

Figure 12: Measurements of the dipole equatorial component of Auger [52] and KASCADE-Grande [53].

In paper [52], the Auger collaboration reported the results of two Rayleigh analyses in the right ascension and azimuth angles reinforcing a previous hint of a dipole anisotropy above $4 \times 10^{18} \mathrm{eV}$. The analysis benefits from the inclusion of the inclined events at large zenith angles allowing a significative increase of the covered sky up to $85 \%$. At the highest energies, above $8 \times 10^{18} \mathrm{eV}$ the large-scale anisotropy has a significance exceeding $4 \sigma$. In the lower energy bin (4-8 EeV) it is less significant. The coordinates of the reconstructed dipole (right ascension, declination) are $(\alpha, \delta)=\left(95^{\circ} \pm 13^{\circ},-39^{\circ} \pm 13^{\circ}\right)$ and $(\alpha, \delta)=\left(15^{\circ} \pm 115^{\circ},-81^{\circ} \pm 17^{\circ}\right)$ at the higher and lower energies respectively. A summary of the measurements of the equatorial amplitudes and of the phases in the wide energy range covered by Auger is shown in Figure 12. When the dipole is not significative, an upper limit is reported. The measurements presented by the KASCADE-Grande collaboration [53] are also shown in the same plot.

The large-scale distribution of arrival directions of cosmic rays has been also studied by combining the data of Auger and Telescope Array [54]. Thanks to the full-sky coverage, it has been possible to report the first angular power spectrum of cosmic rays above $10^{19} \mathrm{eV}$. The data show a significative dipole amplitude and no deviations from isotropy at smaller angular scales.

The Auger collaboration has also reported the results of a search for intermediate scale anisotropies [55]. The analysis includes inclined showers allowing it to select 602 events above $4 \times 10^{19}$ eV. Cross-correlations with complementary catalogs which contain sources within $200 \mathrm{Mpc}$ give results compatible with isotropic expectations. The minima of the penalized probabilities in these searches are at the level of $1 \%$. One minimum is obtained selecting arrival directions around the Cen A radio galaxy. Other tests based on auto-correlation and on the search of excesses in circular windows along the sky yield negative results.

Another search of energy-dependent patterns in the arrival directions of cosmic rays detected by Auger gave negative results [56]. These searches are important since they could give information on galactic and extra-galactic magnetic fields.

The Telescope Array collaboration has reported an update of the search for anisotropies including 2 additional years of data taking, for a total of 7 years [57]. Distributions of the events in right ascension and declination in both equatorial and supergalactic coordinate systems show that there is a moderate deviation from isotropy for the 83 events selected with energies larger than $5.7 \times 10^{19} \mathrm{eV}$, while they are fully compatible with isotropy at lower energies. A similar result is 
obtained by calculating the autocorrelation function.
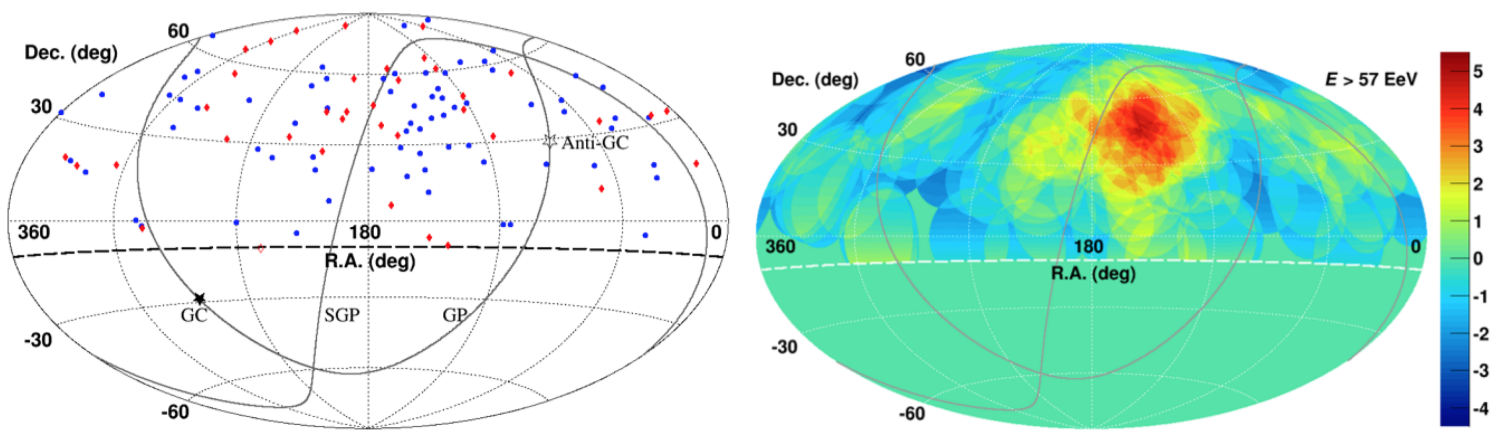

Figure 13: Left panel: sky map in equatorial coordinates of the TA events with energy $>5.7 \times 10^{19}$. The events collected in the first 5 years of data taking are in blue while the ones collected in the last 2 years are in red. Right panel: significance map for the full data sample. The hot spot analysis is described in [57, 58].

The most important result reported by TA concerns the update on the so called hot spot, an angular region of radius $20^{\circ}$ in the direction of $\left(\alpha=148.4^{\circ}, \delta=44.5^{\circ}\right.$ - equatorial coordinates) where there is an excess of events with $E>5.7 \times 10^{19} \mathrm{eV}[19,57,58]$. The sky map of the 37 events collected in the 2 additional years and of the ones of the previous 5 years already published are shown in the left panel of Figure 13. In the first 5 years the number of events in the hot spot is 19 out of a total of 72 , while the expected number of events assuming an isotropic distribution of the arrival directions is 4.49 . The post-trial probability of this excess is $3.7 \times 10^{-4}$ corresponding to $3.4 \sigma$ [59]. In the 37 events of the two additional years, 4 events has been found in the hot spot while 2.31 are expected from isotropy. The probability of having this excess is about $20 \%$. Repeating the analysis presented in [59] over the full dataset of 7 years gives results compatible with the ones already published, with a post-trial significance of $3.4 \sigma$. The significance map for the full data set using $20^{\circ}$ oversampling radius is shown in the right panel of Figure 13.

The center of the hot spot is located at about $17^{\circ}$ from the supergalactic plane, in the vicinity of the Ursa Major cluster. Several potential candidates have been suggested as the source of the hot spot $[58,60]$ but the current statistics are too low to draw any conclusion. Moreover the analysis is complicated by the big uncertainties on the deflections of the cosmic rays during their propagation in the galactic and extra-galactic magnetic fields.

An interesting new approach to search for anisotropy signals is to study the shape of the energy spectrum in different region of the sky. The TA collaboration has found that the spectrum in the sky areas with less nearby objects has a steeper suppression than in those with a larger density of these sources [61]. An example of this study is shown in the left panel of Figure 14, where the region with more objects is the one within $30^{\circ}$ from the super galactic plane. This result is perhaps not surprising since this region partially overlaps with the hot spot where there is an excess of events at the highest energies. However it is interesting to note that a spectrum with a steeper suppression is in better agreement with the one measured by Auger (see Figure 6). The Auger collaboration has performed a similar study presenting, for the first time, the spectrum in different declination bands (see right panel of Figure 14) [20, 21]. As expected, the ratio of the corresponding sub-spectra is in agreement with the dipole anisotropy reported in [52] and therefore there is no evidence of a less steep suppression at higher declinations, toward the Northern Hemisphere seen by TA. 

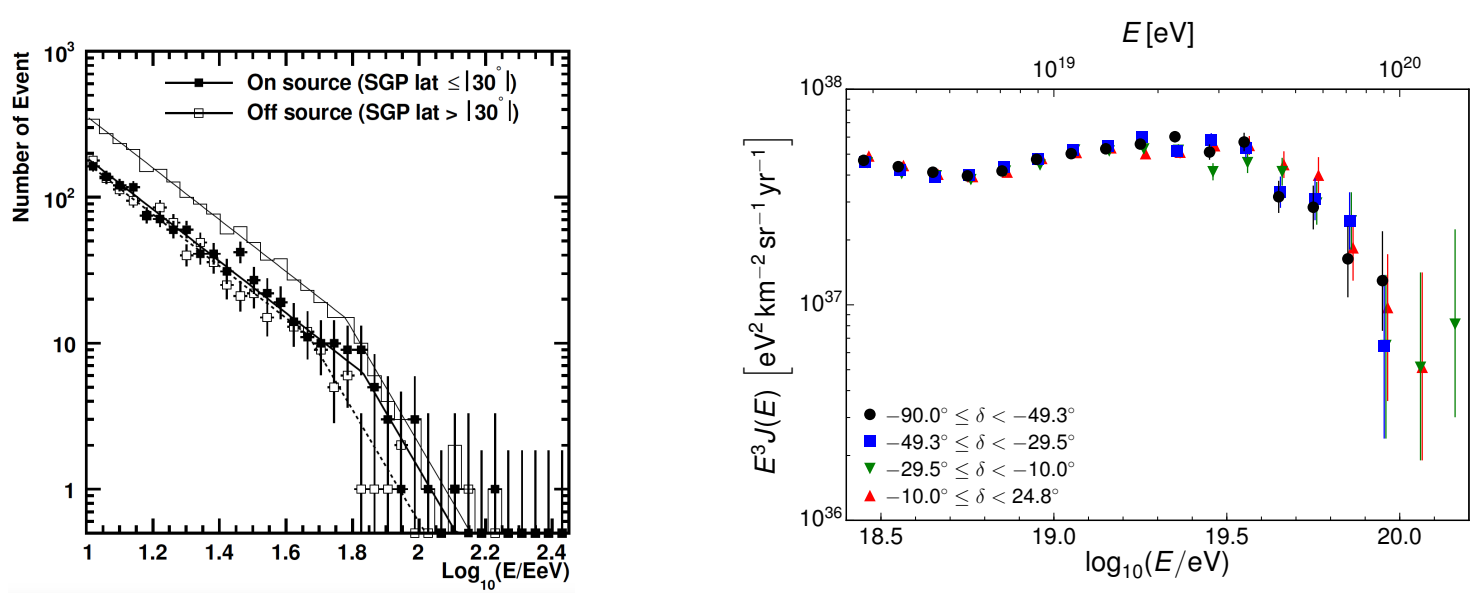

Figure 14: Energy distributions of TA events within $30^{\circ}$ from the super galactic plane and outside this region [61] (left) and Auger energy spectrum in different declination bands [20, 21] (right).

These new approaches to investigate the anisotropy are certainly intriguing because they could explain the difference at the highest energies between the Auger and TA energy spectra. On the other hand, we should remember the big uncertainties affecting the spectrum in the suppression region. The steepness and limited statistics require a very good control of the unfolding of the observed spectrum, that is the procedure of removing the effect of resolution. The uncertainty related to the energy scale is also amplified by the large value of the spectral index. It is clear that a definitive conclusion could be attained only by keeping well under control the systematic uncertainties. A continuous effort is being made by both the collaborations to understand their detector. See papers [62, 63, 64] for Auger and [65, 66] for TA. Particular interesting is paper [65]. The energy calibration of the fluorescence telescopes have been cross-checked using the Electron Light Source (ELS), an electron linear accelerator installed on the TA site at $100 \mathrm{~m}$ from one fluorescence detector station at Black Rock Mesa. The accelerator provides a test of the absolute calibration of the telescopes and of the fluorescence yield used in the analysis of the data. The results of the test are really interesting also because they deal with a comparative test of the different fluorescence yeld models used by TA and Auger.

\section{Hadronic interactions}

One of the hottest topics addressed at the conference are the models describing the hadronic interactions used in the simulation of extensive air showers. The problem is that many interactions in a shower are at energies above those explored by existing accelerators ${ }^{4}$. At these energies, the hadronic multiparticle productions have to be parametrized by phenomenological models because they cannot be calculated from first principles using QCD theory, which is not perturbative at low transverse momenta. A review of the current status of the field has been presented in the invited talk [67].

As already adressed in the previous sections, the hadronic interaction models have a big impact in the interpretation of the $X_{\max }$ data and in the determination of the energy scale of the ground

\footnotetext{
${ }^{4} \mathrm{The} 14 \mathrm{TeV}$ c.m.s. energy of the proton-proton collisions at LHC corresponds to $10^{17} \mathrm{eV}$ in the lab system.
} 
arrays. The muon content of the showers is another observable very sensitive to these models. A measurement of the muon shower size has been presented by the Auger Collaboration [68]. The analysis uses a sample of high quality hybrid events inclined at large zenith angles. In these events the signal in the water-Cherenkov stations of the surface array is dominated by muons since the electromagnetic component is largely absorbed by the atmosphere. The measured average muon content per shower energy as a function of the shower energy is compared with the models in the left panel of Figure 15. There is some tension between data and models. The muon size in the data is even larger than the one predicted for iron when, from the $X_{\max }$ measurements, one would expect an average logarithmic mass between proton and iron [25]. This tension is further underlined by the measurement of the average logarithmic muon content as a function of the average $X_{\max }$ at $10^{19}$ $\mathrm{eV}$ shown in the right panel of Figure 15.
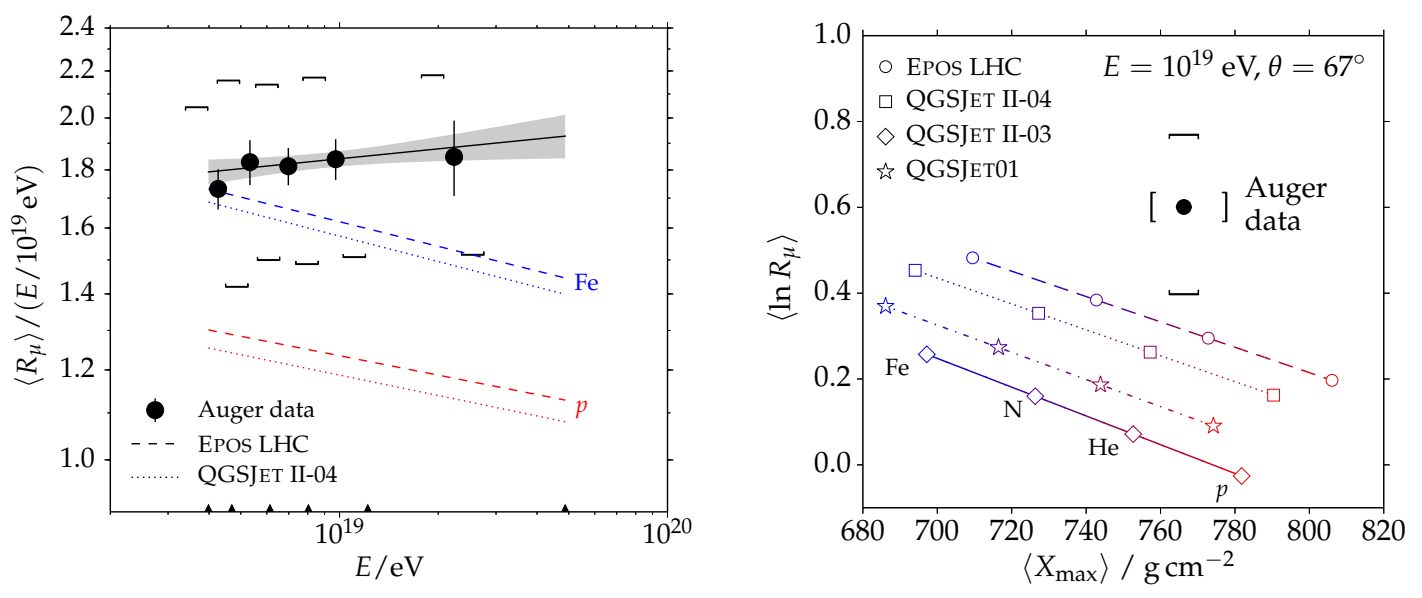

Figure 15: Measurements of the muon content of extensive air showers by the Auger collaboration [68].

Looking at the correlation between the muon size and $X_{\max }$ predicted by the various interaction models, one can perceive the big impact of the LHC measurements (EPOS-LHC and QGSJetII-04 are the only models tuned with the LHC data). The current status and the perspective of the experiments measuring hadronic interactions at the LHC have been the subject of many presentations. Several topics have been addressed, the measurements of the proton-proton collision cross-sections and of the charged particle pseudorapidity distributions by TOTEM [69], the measurements of proton-lead cross-section from CMS [70] and of the production of neutral particles $\left(\gamma, \pi^{0}\right.$ and neutrons) in the very forward region by LHCf [71]. A new important measurement of the multiplicity of the $\rho^{0}$ meson in pion-carbon interactions has been presented by the NA61/SHINE collaboration [72]. They have found that it is underestimated by all hadronic interaction models.

The $\rho^{0}$ production plays a crucial role in the shower development since this meson decays to $\pi^{+} \pi^{-}$. Therefore changing the relative weight of the $\rho^{0}$ and $\pi^{0}$ multiplicities one can change the relative weight of the muon and electromagnetic component of the shower. This kind of tuning is the main modification implemented in a new preliminary version of the event generator Sybill [73].

NA61 has the big advantage of being a fixed target experiment and therefore it can easily cover the very forward region which is the most relevant for shower development. However the 
measurements are limited by the maximum momentum $(350 \mathrm{GeV})$ of the secondary beams of the SPS. As discussed in [74], a big step forward in the understanding of the extensive air showers could be done by realizing a fixed target experiment that uses the beam of the LHC collider.

Measurements like the ones of Auger have triggered a new line of research in which the data from cosmic ray experiments are used to constrain the hadronic models. For example, a new analysis strategy to find an optimal global description of the underlying physics using accelerator and ultra-high energy cosmic ray data has been discussed in [75].

Interesting is also the work presented in [76] in which the hadronic model implement in EPOS is modified in order to describe consistently all Auger mass composition measurements. The problem of EPOS is that it predicts too large values of the depth at which the maximum number of muons are produced. The prediction for iron is larger than the one measured by Auger [68]. That would imply a mass composition with elements heavier than iron. It has been shown that this inconsistency can be eliminated by reducing the elasticity in pion-air interactions by only $10 \%$.

Other experiments have presented measurements relevant for the understanding of the hadronic interaction models. The IceCube collaboration has shown that the muon density at $600 \mathrm{~m}$ measured with IceTop is in good agreement with the predictions of Sybill [77]. KASCADE-Grande finds an inconsistency between data and models in the muon attenuation length [78]. Further tests could be done using measurements of the rate of high muon multiplicity events by ALICE at CERN [79].

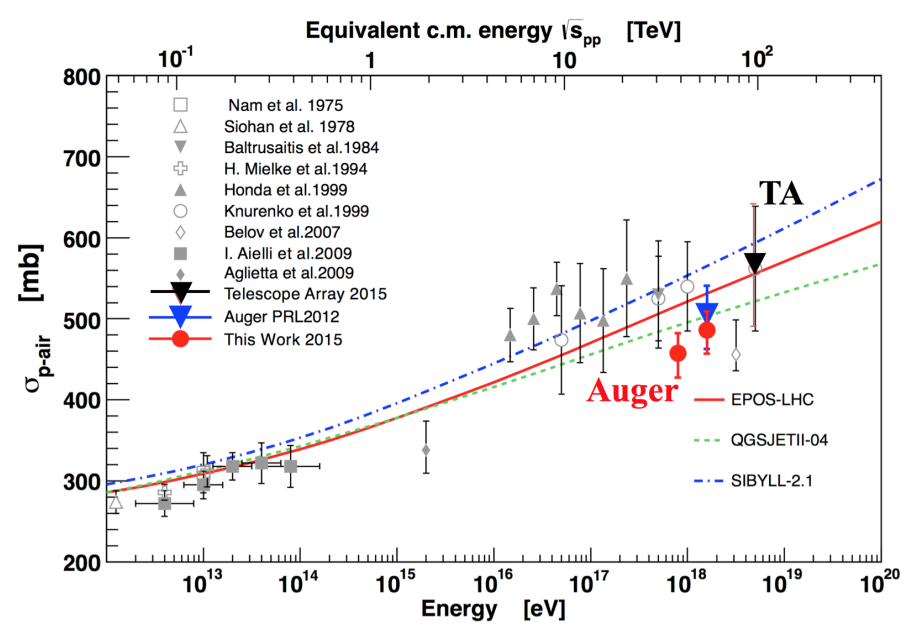

Figure 16: Measurement of the proton-air collision cross section from Auger [80] (red) and TA [81] (black).

Auger and TA have presented their new measurements of the proton-air collision cross section using the data of the fluorescence telescopes [80,81]. The two collaborations use different analysis methods but the underlying information is the same: the attenuation length of proton air showers derived from the $X_{\max }$ distribution is an observable that is strictly correlated with the cross-section of inelastic proton-air collisions. The measured cross-sections are shown in Figure 16. In order to reduce possible biases arising from a contamination of nuclei heavier than protons, the Auger measurements are limited to energies below $10^{18.5} \mathrm{eV}$.

Other analyses that use the measurements of the fluorescence telescopes to test the models have been developed by Auger [82] and TA [83]. In fact, the average shape of the longitudinal profiles is to some extent sensitive to the inelasticity and multiplicity in the early part of the shower. These 
studies are very interesting but, given the rather modest sensitivity of the observables, a detailed understanding of the not trivial systematic uncertainties of the measurements is needed.

\section{The radio detection technique}

The field of the radio detection of cosmic ray air showers has had an impressive growth of interest in the past decade. More than 40 contributions have been presented at this conference, in line with the previous ICRC conference in 2013. The interest arises from the fact that the radio signals are generated by the electromagnetic component of the shower and is integrated over the full shower development. Therefore they can provide a calorimetric measurement of the shower energy with a duty cycle of $100 \%$, overcoming the limitations of the current detection techniques.

The main mechanism of radio emission is the interaction of secondary electrons and positrons with the Earth's magnetic field ("geosynchrotron emission"). A secondary contribution arises from the negative charge excess that follows as the moving cascade grows ("Askaryan emission").

There are currently several detectors taking data. The typical working frequencies range from a few to hundreds of MHz. The Low Frequency Array (LOFAR) [84] located in the Netherlands is a small but very dense array of antennas which allows a very precise measurement of the radio footprint at the ground. In LOFAR, scintillator detectors are used for triggering purposes [85]. The Auger Engineering Radio Array (AERA) is instead a low density array covering an area of $17 \mathrm{~km}^{2}$ at the Auger site [86]. The Tunka Radio Extension (Tunka-Rex) [87] is an another array distributed over $3 \mathrm{~km}^{2}$ and it is an extension of Tunka-133, an air-Cherenkov detector in Siberia. CODALEMA is an experiment located at the Nançay Radio Observatory [88] operating over a unique and very wide frequency band (from 2 to $200 \mathrm{MHz}$ ). LOPES is a pioneering experiment, no longer operating, that was installed at the site of KASCADE-Grande [89].

Several advances in the development of the radio detection technique have been reported at this conference. Many works demonstrate that the underlying emission mechanism is well understood. The contribution to the total signal of the "Askaryan emission" has been measured to be at the level of $10 \%$ [90], as expected from the theory. The effort of the SLAC-T510 experiment at the SLAC National Accelerator Laboratory is remarkable. Its aim is to validate the model predictions studying the radio emission produced by an electron beam which collides in an high density polyethylene target placed in a variable magnetic field. The results have been presented in $[91,92,93]$. Notable is also the re-analysis of the LOPES data based on a new calibration of the antennas [89], showing good agreement between the amplitude of the radio signal recorded in coincidence with the air showers detected by the KASCADE-Grande array and its theoretical prediction made with the CoREAS simulation code [89, 94].

Most of the presentations dealt with the reconstruction of the shower observables. The first step of the reconstruction is the determination of the lateral density function. The two components of the radio signal have different polarisations and therefore they add constructively or destructively depending by the observer position, producing an asymmetric footprint at ground. Besides the complexity of the problem, LOFAR has demonstrated that the two dimensional lateral density function (LDF) could be described by an analytical parameterisation [95].

The amplitude of the LDF provides an estimator of the shower energy. AERA measures 15.8 $\mathrm{MeV}$ of radiation energy between 30 and $80 \mathrm{MHz}$ for a $1 \mathrm{EeV}$ air shower arriving perpendicular to 
the geomagnetic field at the Auger site [96]. The signal scales quadratically with the shower energy as expected for coherent emission. The analysis is based on a comparison of the radio signals with the data of the Auger surface array and it demonstrates that the radio technique provides a shower energy with a resolution even better than $20 \%$.

The slope of the LDF provides information on the shower evolution. In LOFAR $X_{\max }$ is determined by performing a fit to the measured LDF against the predictions of the CoREAS simulations [97]. The high density of the array and the accurate calibration of the antennas [98] allow one to get a resolution on $X_{\max }$ of about $15 \mathrm{~g} / \mathrm{cm}^{2}$, comparable to the one of the fluorescence telescopes. A similar method has been developed in CODALEMA [99]. Another reconstruction technique aiming to get information on $X_{\max }$ is described in [100]. A remarkable proof that the radio detectors can provide $X_{\max }$ has been provided by Tunka-Rex. An analysis of the showers detected in coincidence with the Tunka-133 air Cherenkov detectors shows a good agreement between the $X_{\max }$ estimations of the two detectors [87]. The calibration of the Tunka-Rex antennas is described in [101].
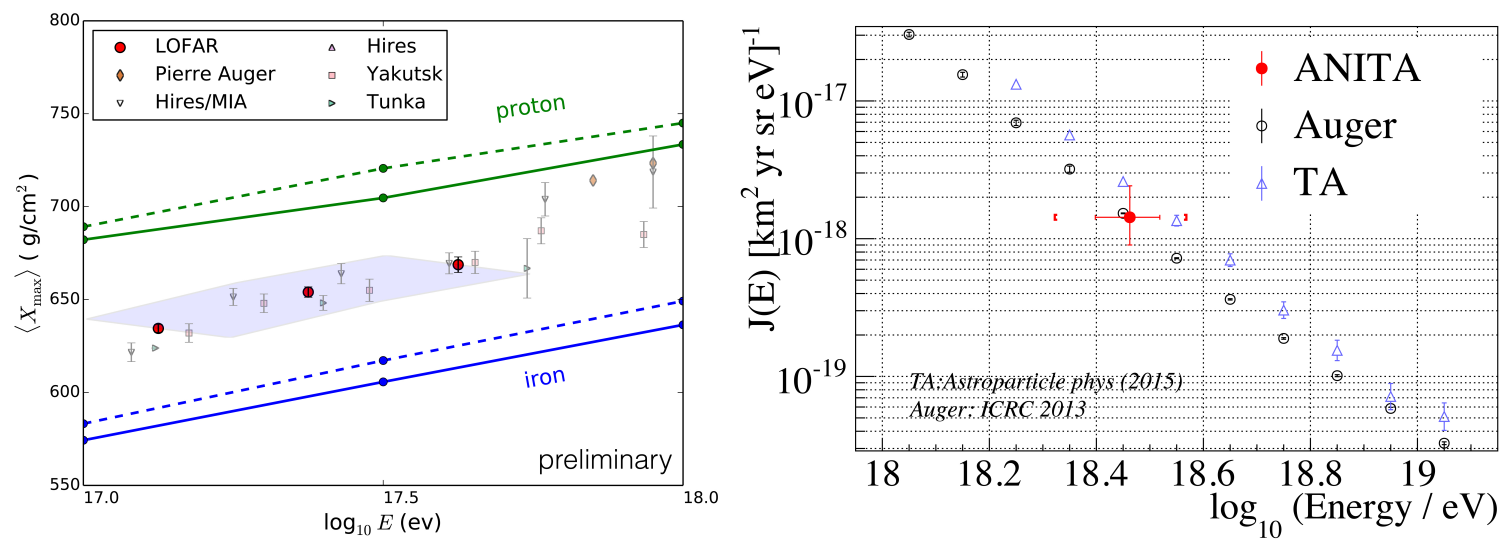

Figure 17: Left panel: first measurement of the mean depth of the shower maximum by LOFAR [84, 102]. Right panel: energy spectrum measured by ANITA [104].

A first measurement of the mean depth of the shower maximum by LOFAR using a sample of 118 showers is shown in the left panel of Figure 17 [84, 102]. Another measurement of the mean $X_{\max }$ based on the radio array installed in the Yakutsk site was presented in [103].

In the right panel of Figure 17, the energy spectrum of cosmic rays measured by ANITA is shown [104]. Although ANITA is a balloon radio detector conceived to measure impulsive radiation from ultra-high energy neutrino interactions in the ice, it has detected several cosmic ray showers. The interpretation of the signal reflected off the ice has been done using a new version of the ZHAireS simulation code [105] and has allowed an estimation of the energy spectrum. The interest of this detection technique is the large atmospheric volume that could be monitored using only few radio receivers. TAROGE [106] is another project that would implement a similar technique looking to the radiation reflected from the ocean.

Although the main aim of the radio detectors is the detection of atmospheric showers, the same detectors can be potentially used also to study the particles above $10^{21} \mathrm{eV}$ using the lunar Askaryan technique [107]. It is also interesting how the measurements during thunderstorms could provide information on the atmospheric electric fields along the shower axis [108]. 
Other alternative radio detection techniques of atmospheric showers are not promising. A very stringent upper limit on the radar cross section, significantly below the value needed to detect cosmic rays, has been put with the TARA experiment operated at the TA site [109]. Another test using the TA ELS gave negative results [110] and also theoretical calculations are rather pessimistics [111]. Also the detection of the molecular bremsstrahlung radiation produced in air showers is not promising. Other than the studies previously performed by the Auger collaboration [112], no signals have been detected using the ELS of TA [113] and theoretical models of the emission process predict an intensity much lower than the one needed to make the detection technique feasible [114].

\section{Future experiments}

It has been demonstrated that the radio detection technique provides accurate measurements of shower observables, but with the strong limitation arising from the rather small footprint at ground of the radio signals. This means that the technique is not suitable for the detection of cosmic rays at the very high energies that require large arrays, unless one restricts the field of view to inclined showers, whose footprint is significantly larger [86]. A very attractive proposal for using the future world's largest radio telescope Square Kilometre Array (SKA) to detect cosmic rays has been discussed in [115]. In SKA 60,000 antennas will be deployed in an area of $1 \mathrm{~km}^{2}$, offering a unique opportunity to measure cosmic rays between $10^{16}$ and $10^{18} \mathrm{eV}$ with very good precision. Additional detectors will be installed to provide the trigger. The opportunity to use SKA for the application of the lunar Askaryan technique has been discussed in [116].

The Large High Altitude Air Shower Observatory (LHAASO) [117] is another detector designed to make accurate measurements of the showers in the energy region of the knees. It consists of instrumenting with different kinds of detectors an area of $1 \mathrm{~km}^{2}$ in the Sichuan province, China. The installation of the detector is expected to start in 2016 and to end in 2020.

The potentiality of a future IceCube-Gen2, extending the current $1 \mathrm{~km}^{2}$ IceTop area by a factor 5-10 has been discussed in [118].

At the highest energies the Auger Collaboration has presented AugerPrime [119], the upgrade of the detector which will be in operation from 2018 until 2024. The key element of AugerPrime will be the installation of a new detector consisting of a plastic scintillator plane above each of the existing water-Cherenkov detectors. The idea is to combine the measurements of the scintillators and of the Cherenkov stations, which are sensitive in a different way to the electromagnetic particles and to the muons, to extract the electromagnetic and muonic component of the showers $[119,120]$. In this way composition-sensitive information can be obtained in the region of the cut-off, where the measurement of $X_{\max }$ by the fluorescence telescopes are not available due to the reduced duty cycle of $15 \%$ of the detector.

The Telescope Array Collaboration has presented TA $\times 4$ [121], the extension of the current array from the actual $700 \mathrm{~km}^{2}$ to approximately $3,000 \mathrm{~km}^{2}$, the size of the Auger array. The extension was approved in Japan in April 2015 and the deployment of the additional scintillators will begin very soon and is expected to end in December 2017. A proposal to build new fluorescence telescopes to cover the extended array has been submitted in the US. TA $\times 4$ will allow to increment significantly the statistics in the region of the cut-off, where the hot spot has been observed. 
The aim of the JEM-EUSO mission [122] is to study the cosmic rays at the highest energies, up to $\sim 10^{20} \mathrm{eV}$ [123]. The mission consists of installing a wide field of view fluorescence telescope onboard of the International Space Station (ISS). The program to define the final configuration of the mission is rather complex. Several improvements have been foreseen to allow the use of Space-X Dragon spacecraft. The KLYPVE mission attached to the Russian module of the ISS is in the stage of final definition. The EUSO-Balloon flew on board a stratospheric balloon [124] and EUSO-TA [125], at the Telescope Array site, are two prototypes of the detector which have already operated. The future pathfinder Mini-EUSO mission onboard the ISS has been discussed in [126].

Several ideas on the development of new detectors have been presented. We mention FAST, a Fluorescence detector Array of Single-pixel Telescopes that could be an economical solution to use the fluorescence detection technique in experiments covering very large area [127].

\section{Outlook}

The large number of contributions dealing with the cosmic rays studied through the detection of atmospheric showers demonstrates an extraordinary vitality of the scientific community in this field, with many new measurements and new ideas on instruments and analyses.

We may conclude this report with the following summary.

- The features of the energy spectrum have been confirmed with improved statistics. The existence of the low energy ankle at $\sim 10^{16} \mathrm{eV}$ and of the second knee at $\sim 10^{17} \mathrm{eV}$ have been confirmed by IceCube [13], Yakutsk [11], KASKADE-Grande [10] and the TALE telescopes of TA [16]. The measurements of the position of the ankle at $5 \times 10^{18} \mathrm{eV}$ by Auger $[20,21]$ and TA $[19,16]$ are in excellent agreement. The suppression at the highest energies is observed by TA and by Auger, the latter with an unprecedented statistics.

- The $\mathrm{p} / \mathrm{He}$ knee observed by the ARGO-YBJ collaboration at $\sim 700 \mathrm{TeV}[3,5,4]$ deserves attention since it is in tension with the paradigm that explains the first knee at $\sim 3 \times 10^{15} \mathrm{eV}$ and the second one with a rigidity mechanism related to maximum acceleration at the sources or maximum energy of Galactic magnetic confinement for protons and highest-Z Galactic cosmic rays. It is important for the ARGO-YBJ results to be confirmed by other experiments.

- The IceCube [13] and Yakutsk [24] data shows that the composition becomes heavier from the first knee to the second knee. Above $\sim 10^{17} \mathrm{eV}$ the composition becomes lighter as confirmed by Yakutsk and the new measurement by Auger using the HEAT telescopes [25]. The Auger data show another change of the trend at an energy slightly smaller than the one of the ankle, with the composition that becomes heavier above this energy. The TA $X_{\max }$ data [29] suffer from less statistics and of a higher energy threshold than Auger, not allowing them to see the change of the trend. The $X_{\max }$ measurements of Auger and TA are in agreement within the quoted systematic uncertainties [30].

- The inference on the mass composition from the cosmic rays data suffers from the large, and ultimately unknown, uncertainties on the hadronic interaction models. There is an impressive effort to make the models more reliable by using the LHC data and looking at the consistency between models and measurements done by cosmic rays experiments [67]. Remarkable is the underestimation of the $\rho^{0}$ production cross section reported by NA61/SHINE [72] that would 
imply an higher muon content of the showers, in better agreement with the measurements of Auger [68].

- The interpretation of the ankle is not trivial. The "dip" scenario [39] requires an almost pure composition of extragalactic protons and it seems to be in tension with the Auger data that suggest that there could be a mixed composition at these energies. Other different interpretations of the ankle have been presented at this conference, demonstrating that the scientific community is still far away from reaching a consensus on how to explain this feature.

- According to the analyses reported by Auger [36] and TA [38], the interpretation of the suppression at the highest energies turns around two scenarios: a maximum acceleration at the sources and a propagation effect. The current situation seems rather controversial. In his model, TA assumes a pure proton composition that it is clearly in tension with the Auger data. On the other hand, under the assumptions made in his model, Auger seems to favour a maximum acceleration scenario when the most natural interpretation of the suppression would be a GZK mechanism [37]. In fact the GZK suppression is a well-known process, predicted a long time ago, that occurs for protons and iron at a similar energies, and therefore that could explain the energy spectrum even if the flux is not made by only protons.

- The level of isotropy of the arrival directions of cosmic rays at about $10^{18} \mathrm{eV}$ suggests that these particles are of extragalactic origin. At higher energies Auger observes a dipole [52, 54] while TA observes the hot spot at the cut-off [58]. The moderate level of anisotropy could be due to the presence of nuclei heavier than protons, that are expected to suffer big deflections in the galactic and extra-galactic magnetic fields, thus not allowing the identification of the sources.

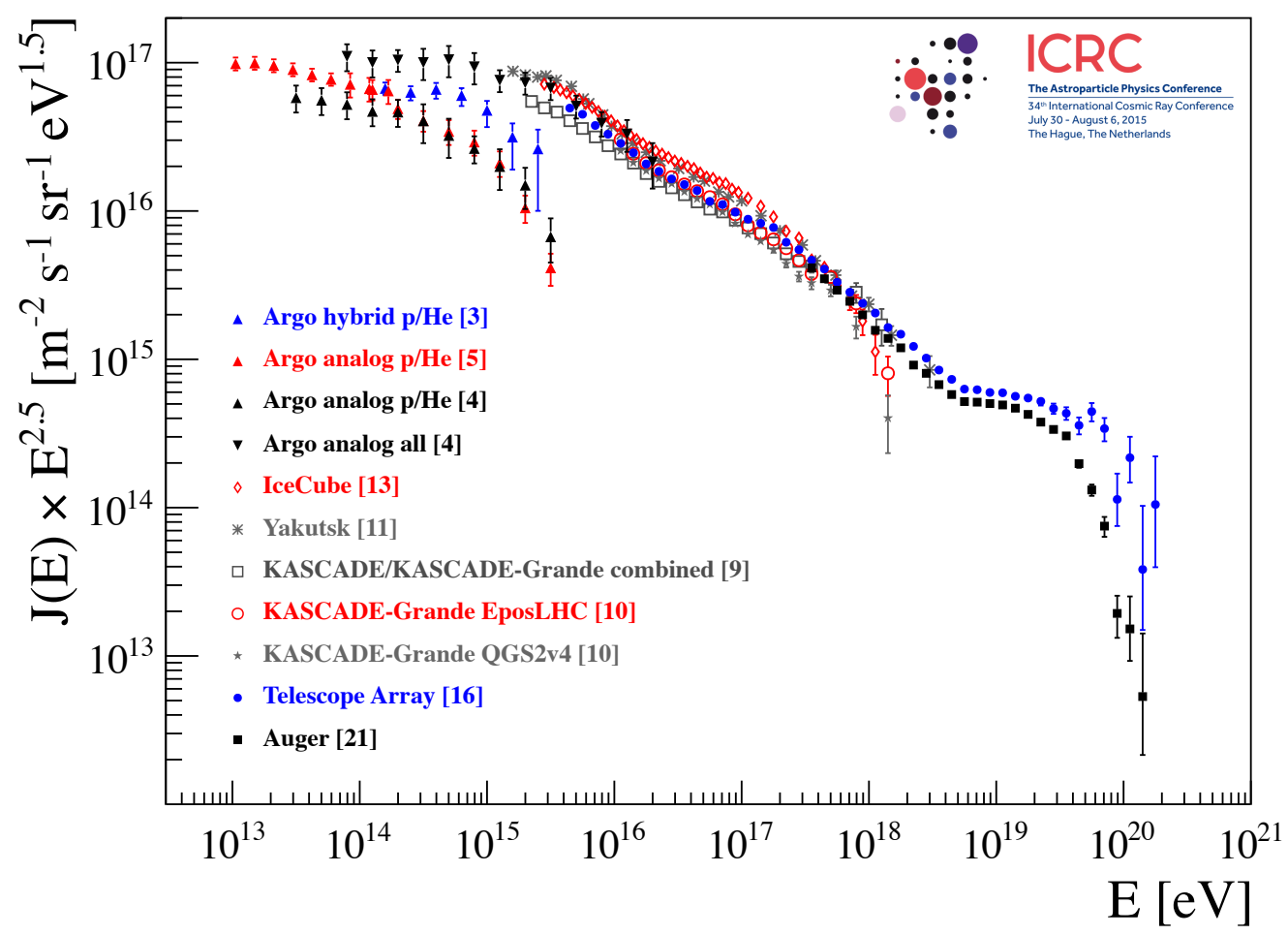

Figure 18: Energy spectra presented at the 34th International Cosmic Ray Conference. 
From the above summary it is clear that the future projects or upgrades of the existing detectors go in the right direction to answer to the many open questions on the ultra-high energy cosmic rays. The TA array will be extended by a factor 4 [121], reaching the same size of Auger. The upgrade of Auger [119] will allow to make mass composition measurements in the region of the suppression. In few years, Auger+TAx4 will offer the unique opportunity to have a fulll sky coverage with a total extension of $6000 \mathrm{~km}^{2}$. On a longer time scale, JEM-EUSO [122] will accumulate further statistics with an annual exposure equal to 5 times the one of Auger+TAx4. Future experiments at lower energies will address other important issues like the transition from the Galactic to extragalactic cosmic rays. As discussed in Sec. 5, the experiments at LHC will allow a more accurate understanding of the extensive air showers.

We conclude this report showing in Figure 18 all the energy spectra presented at this conference and thanking the authors for providing the data.

\section{References}

[1] P. Maestro, Cosmic Rays: direct measurements - Rapporteur Report, PoS (ICRC2015) 016.

[2] G. Matthiae and V. Verzi, Riv. Nuovo Cimento, 38 (2015) 73 and references therein.

[3] S. S. Zhang and Z. Cao, for the LHAASO and ARGO-YBJ Collaborations, PoS (ICRC2015) 261.

[4] I. De Mitri et al., for the ARGO-YBJ Collaboration, PoS (ICRC2015) 366.

[5] S. M. Mari and P. Montini, for the ARGO-YBJ Collaboration, PoS (ICRC2015) 371.

[6] P. Di Sciascio, for the ARGO-YBJ Collaboration, private communication.

[7] J. Huang, for the Tibet AS $\gamma$ Collaboration, PoS (ICRC2015) 421.

[8] A. Haungs, for the KASCADE-Grande Collaboration, PoS (ICRC2015) 278.

[9] S. Schoo, for the KASCADE-Grande Collaboration, PoS (ICRC2015) 263.

[10] M. Bertaina, for the KASCADE-Grande Collaboration, PoS (ICRC2015) 359.

[11] I. Petrov et al., PoS (ICRC2015) 252.

[12] T. Karg, for the IceCube Collaboration, PoS (ICRC2015) 365.

[13] K. Rawlins and T. Feusels, for the IceCube Collaboration, PoS (ICRC2015) 334.

[14] T. AbuZayyad, for the Telescope Array Collaboration, PoS (ICRC2015) 422.

[15] Z. Zundel, for the Telescope Array Collaboration, PoS (ICRC2015) 445.

[16] D. Ivanov, for the Telescope Array Collaboration, PoS (ICRC2015) 349.

[17] T. Fujii, for the Telescope Array Collaboration, PoS (ICRC2015) 320.

[18] D. Ikeda, for the Telescope Array Collaboration, PoS (ICRC2015) 362.

[19] C. Jui, for the Telescope Array Collaboration, Summary of Results from the Telescope Array Experiment, PoS (ICRC2015) 035.

[20] P. Ghia, for the Pierre Auger Collaboration, Highlights from the Pierre Auger Observatory, PoS (ICRC2015) 034.

[21] I. Valiño, for the Pierre Auger Collaboration, PoS (ICRC2015) 271.

[22] V. Berezinsky et al., Phys. Rev. D, 74 (2006) 043005. 
[23] I. Mariş et al., for the Pierre Auger and Telescope Array Collaborations, Proceedings of the International Symposium UHECR2014, in preparation, Springdale U.S.A. (2014).

[24] I. Petrov et al., PoS (ICRC2015) 254.

[25] A. Porcelli, for the Pierre Auger Collaboration, PoS (ICRC2015) 420.

[26] J. P. Lundquist, for the Telescope Array Collaboration, PoS (ICRC2015) 441.

[27] J. P. Lundquist, for the Telescope Array Collaboration, PoS (ICRC2015) 442.

[28] T. Stroman, for the Telescope Array Collaboration, PoS (ICRC2015) 361.

[29] J. Belz, for the Telescope Array Collaboration, PoS (ICRC2015) 351.

[30] M. Unger, for the Pierre Auger and Telescope Array Collaborations, PoS (ICRC2015) 307.

[31] C. Bleve, for the Pierre Auger Collaboration, PoS (ICRC2015) 1103.

[32] G. I. Rubtsov et al., for the Telescope Array Collaboration, PoS (ICRC2015) 331.

[33] K. Yamazaki et al., for the Telescope Array Collaboration, PoS (ICRC2015) 356.

[34] Z. Feng, for the KASCADE-Grande Collaboration, PoS (ICRC2015) 823.

[35] T. Fujii, for the Pierre Auger Collaboration, PoS (ICRC2015) 319.

[36] A. di Matteo, for the Pierre Auger Collaboration, PoS (ICRC2015) 249.

[37] K. Greisen, Phys. Rev. Lett. 16 (1966) 183; G. T. Zatsepin and V. A. Kuz'min, Sov. Phys. JETP Lett. 4 (1966) 114.

[38] E. Kido and O. E. Kalashev, for the Telescope Array Collaboration, PoS (ICRC2015) 258.

[39] V. Berezinsky et al., Phys. Rev. D 74 (2006) 043005.

[40] A. Yushkov, for the Pierre Auger Collaboration, PoS (ICRC2015) 335.

[41] D. Allard et al., Astronomy and Astrophysics 443 (2005) L29.

[42] D. Ivanov et al., for the Telescope Array Collaboration, PoS (ICRC2015) 350.

[43] N. Globus et al., PoS (ICRC2015) 515.

[44] G. Farrar et al., PoS (ICRC2015) 513.

[45] D. W. Fiorino, for the HAWC Collaboration, PoS (ICRC2015) 241.

[46] S. Westerhoff, for the IceCube Collaboration, PoS (ICRC2015) 274.

[47] M. Sutherland, for the IceCube Collaboration, PoS (ICRC2015) 250.

[48] J. C. Díaz-Vélez et al., for the HAWC and IceCube Collaborations, PoS (ICRC2015) 444.

[49] Z. Feng, for the Tibet AS $\gamma$ Collaboration, PoS (ICRC2015) 355.

[50] K. Munakata, for the Tibet AS $\gamma$ Collaboration, PoS (ICRC2015) 279.

[51] R. Iuppa et al., for the ARGO-YBJ Collaboration, PoS (ICRC2015) 290.

[52] I. Al Samarai, for the Pierre Auger Collaboration, PoS (ICRC2015) 372.

[53] A. Chiavassa, for the KASCADE-Grande Collaboration, PoS (ICRC2015) 281.

[54] O. Deligny, for the Pierre Auger and Telescope Array Collaborations, PoS (ICRC2015) 395.

[55] J. Aublin, for the Pierre Auger Collaboration, PoS (ICRC2015) 310.

[56] T. Winchen, for the Pierre Auger Collaboration, PoS (ICRC2015) 294. 
[57] P. Tinyakov et al., for the Telescope Array Collaboration, PoS (ICRC2015) 326.

[58] K. Kawata et al., for the Telescope Array Collaboration, PoS (ICRC2015) 276.

[59] R. U. Abbasi et al., the Telescope Array Collaboration, Astrophys. J. 790 (2014) L21.

[60] H. Haoning et al., PoS (ICRC2015) 325.

[61] T. Nonaka et al., for the Telescope Array Collaboration, PoS (ICRC2015) 384.

[62] G. Salina, for the Pierre Auger Collaboration, PoS (ICRC2015) 594.

[63] C. Medina-Hernandez, for the Pierre Auger Collaboration, PoS (ICRC2015) 624.

[64] P. Assis, for the Pierre Auger Collaboration, PoS (ICRC2015) 620.

[65] B. Shin et al., for the Telescope Array Collaboration, PoS (ICRC2015) 640.

[66] M. Hayashi et al., for the Telescope Array Collaboration, PoS (ICRC2015) 692.

[67] R. Engel, Relations between high-energy particle and cosmic-ray physics, PoS (ICRC2015) 007.

[68] L. Collica, for the Pierre Auger Collaboration, PoS (ICRC2015) 336.

[69] F. S. Cafagna, for the TOTEM Collaboration, PoS (ICRC2015) 312.

[70] C. Baus et al., for the CMS Collaboration, PoS (ICRC2015) 440.

[71] Y. Itow, for the LHCf Collaboration, PoS (ICRC2015) 259.

[72] A. E. Hervé, for the NA61/SHINE Collaboration, PoS (ICRC2015) 330.

[73] R. Engel et al., PoS (ICRC2015) 558.

[74] R. Ulrich et al., PoS (ICRC2015) 407.

[75] C. Baus et al., PoS (ICRC2015) 418.

[76] T. Pierog et al., PoS (ICRC2015) 337.

[77] H. P. Dembinski and J. G. Gonzalez, for the IceCube Collaboration, PoS (ICRC2015) 267.

[78] J. C. Arteaga-Velázquez, for the KASCADE-Grande Collaboration, PoS (ICRC2015) 314.

[79] M. R. Cahuantzi, for the ALICE Collaboration, PoS (ICRC2015) 423.

[80] R. Ulrich, for the Pierre Auger Collaboration, PoS (ICRC2015) 401.

[81] R. Abbasi and J. Belz, for the Telescope Array Collaboration, PoS (ICRC2015) 402.

[82] F. Diogo, for the Pierre Auger Collaboration, PoS (ICRC2015) 413.

[83] D. R. Bergman and T. A. Stroman, for the Telescope Array Collaboration, PoS (ICRC2015) 339.

[84] J. R. Hörandel, Radio detection of Cosmic Rays with LOFAR, PoS (ICRC2015) 033.

[85] S. Thoudam et al., PoS (ICRC2015) 327.

[86] J. Schulz, for the Pierre Auger Collaboration, PoS (ICRC2015) 615.

[87] D. Kostunin, for the Tunka-Rex Collaboration, PoS (ICRC2015) 285.

[88] R. Dallier et al., PoS (ICRC2015) 293.

[89] T. Huege, for the LOPES Collaboration, PoS (ICRC2015) 311.

[90] A. Corstanje et al., PoS (ICRC2015) 396.

[91] K. Belov et al., PoS (ICRC2015) 346. 
[92] S. A. Wissel et al., PoS (ICRC2015) 342.

[93] A. Zilles et al., PoS (ICRC2015) 313.

[94] F. G. Schröder, for the LOPES Collaboration, PoS (ICRC2015) 317.

[95] A. Nelles et al., PoS (ICRC2015) 376.

[96] C. Glaser, for the Pierre Auger Collaboration, PoS (ICRC2015) 364.

[97] S. Buitink et al., PoS (ICRC2015) 369.

[98] J. R. Hörandel et al., PoS (ICRC2015) 662.

[99] F. Gaté et al., PoS (ICRC2015) 397.

[100] L. Rossetto et al., PoS (ICRC2015) 381.

[101] R. Hiller, for the Tunka-Rex Collaboration, PoS (ICRC2015) 573.

[102] S. Buitink et al., PoS (ICRC2015) 368.

[103] I. Petrov et al., PoS (ICRC2015) 255.

[104] H. Schoorlemmer et al., PoS (ICRC2015) 272.

[105] D. García-Fernández et al., PoS (ICRC2015) 266.

[106] J. Nam et al., PoS (ICRC2015) 663.

[107] S. ter Veen et al., PoS (ICRC2015) 684.

[108] T. N. G. Trinh et al., PoS (ICRC2015) 284.

[109] I. Myers and J. Belz, for the Telescope Array Radar and Telescope Array Collaborations, PoS (ICRC2015) 434.

[110] D. Ikeda, for the Telescope Array Collaboration, PoS (ICRC2015) 363.

[111] J. Stasielak et al., PoS (ICRC2015) 316.

[112] A. Haungs, for the Pierre Auger Collaboration, PoS (ICRC2015) 593.

[113] T. Yamamoto et al., for the Telescope Array Collaboration, PoS (ICRC2015) 579.

[114] I. Al Samarai and O. Deligny, PoS (ICRC2015) 417.

[115] T. Huege et al., PoS (ICRC2015) 309.

[116] C. W. James et al., PoS (ICRC2015) 291.

[117] H. He, for the LHAASO Collaboration, PoS (ICRC2015) 1010.

[118] D. Seckel, for the IceCube-Gen2 Collaboration, PoS (ICRC2015) 694.

[119] R. Engel, for the Pierre Auger Collaboration, PoS (ICRC2015) 686.

[120] M. Roth et al., PoS (ICRC2015) 378.

[121] H. Sagawa, for the Telescope Array Collaboration, PoS (ICRC2015) 657.

[122] A. Santangelo et al., for the JEM-EUSO Collaboration, PoS (ICRC2015) 618.

[123] A. V. Olinto et al., for the JEM-EUSO Collaboration, PoS (ICRC2015) 623.

[124] P. von Ballmoos, for the JEM-EUSO Collaboration, PoS (ICRC2015) 322.

[125] M. Casolino et al., for the JEM-EUSO Collaboration, PoS (ICRC2015) 636.

[126] M. Ricci et al., for the JEM-EUSO Collaboration, PoS (ICRC2015) 599.

[127] T. Fujii et al., PoS (ICRC2015) 323. 